\title{
Eliminating Mixed Asymptotics in Obstacle Type Free Boundary Problems
}

\author{
Ivan Blank* \\ Department of Mathematics, Worcester Polytechnic Institute, \\ Worcester, Massachusetts, USA
}

\begin{abstract}
We show a method to eliminate a type of mixed asymptotics in certain free boundary problems, and give two examples of its application. It appears that these problems cannot be handled by the monotonicity formula of Alt et al. [Alt, H. W., Caffarelli, L. A., Friedman, A. (1984). Variational problems with two phases and their free boundaries. Trans. Am. Math. Soc. 282(2):431-461] or by the more recent monotonicity formula of Caffarelli et al. [Caffarelli, L. A., Jerison, D., Kenig, C. E. (2002). Some new monotonicity theorems with applications to free boundary problems. Ann. Math. (2) 155(2):369-404].
\end{abstract}

Key Words: Obstacle problem; Free boundary problem; Eigenvalue problem; Asymptotics; Monotonicity formula; Blow up.

2000 Mathematics Subject Classification: $35 \mathrm{R} 35 ; 35 \mathrm{Q} 72 ; 74 \mathrm{~A} 40$.

*Correspondence: Ivan Blank, Department of Mathematics, Worcester Polytechnic Institute, 100 Institute Road, Worcester, MA 01609, USA; Fax: (508) 831-5824; E-mail: blanki@, wpi.edu. 


\section{INTRODUCTION}

In the obstacle problem one studies the minimum superharmonic function $u$ which lies above a given obstacle, $\varphi$. If we let $w$ denote the height of the solution minus the height of the obstacle, then it is not too hard to show that $w$ satisfies (we will assume that the domain of $w$ contains $B_{1}$ ):

$$
w \geq 0 \quad \text { and } \quad \Delta w(x)=\chi_{\{w>0\}}(x) f(x) \text { in } B_{1} .
$$

Here $f:=-\Delta \varphi$ where $\varphi$ is the original obstacle. For the sake of studying the regularity of the contact set, the nondegeneracy condition

$$
0<\lambda \leq f \leq \Lambda
$$

is usually imposed, and we assume it here. We will also assume that $f$ is continuous at 0 . If $w(0)=\|\nabla w(0)\|=0$, and 0 belongs to the boundary of the set where $w$ vanishes, then one can show that $w$ is universally bounded on $B_{1 / 2}$. (The constant will depend linearly on $\Lambda$, but otherwise it will only depend on the dimension of the space. See Blank, 2001; Brézis and Kinderlehrer, 1973/1974; Caffarelli, 1998 or Caffarelli and Kinderlehrer, 1980 for a proof.) In particular, this universal bound gives enough compactness to guarantee that the quadratic rescaling

$$
w_{\epsilon}(x):=\epsilon^{-2} w(\epsilon x)
$$

will converge to a nontrivial global solution of

$$
w \geq 0 \quad \text { and } \quad \Delta w(x)=\chi_{\{w>0\}}(x) f(0) .
$$

At that point classification of these global blow up limits can be used to study questions of local regularity of the free boundary. (See Blank, 2001 or Caffarelli, 1998 for example.)

Now in a more general setting, if we assume only that $w$ satisfies

$$
|\Delta w| \leq \Lambda \quad \text { and } \quad w(0)=\|\nabla w(0)\|=0
$$

then we will not have a quadratic bound on $w$ at zero and we cannot guarantee that the standard quadratic rescaling will converge. Instead we have only a Zygmund class estimate which yields (assuming $w$ is bounded on $\partial B_{1}$ by $\gamma$ ):

$$
|w(x)| \leq C(n) \Lambda \gamma \|\left. x\right|^{2}(1+|\log (|| x||)|) .
$$

In this situation trying to get useful information from some sort of blow up becomes a much more difficult task. To give an idea of the problem, suppose that $w$ is not bounded by a parabola on any sequence of radii converging to zero, and suppose 
that we do a rescaling of the form

$$
w_{n}(x):=\gamma_{n} w\left(\epsilon_{n} x\right)
$$

where $\epsilon_{n} \downarrow 0$, and $\gamma_{n}$ is chosen to keep some norm of $w_{n}$ fixed. We will call a blow up of this form an inhomogeneous blow up to distinguish it from the more usual quadratic blow up where the $\gamma_{n}$ are exactly $\epsilon_{n}^{-2}$. (For now we may as well assume that we have the sup norm of $w_{n}$ on the unit ball to be fixed at 1.) Because $w$ does not have a quadratic bound, the renormalization will lead to the estimate $\Delta w_{n} \rightarrow 0$ and standard regularity and compactness results allow us to conclude that a subsequence of the $w_{n}$ converges to a harmonic function $W$ on any compact subset of $B_{1}$. The problem is that the norm which we fix at 1 can "concentrate", on $\partial B_{1}$ and we can have $W \equiv 0$. (It is possible that the $w_{n}$ is close to $r^{n} \sin (n \theta)$ for example.)

The goal of this paper is to show how in a couple of examples of free boundary problems there is still a successful blow up argument which leads to a partial classification of the asymptotics of the solution. For a function $w$ which satisfies the assumptions above, we distinguish between three possibilities:

(1) If there exists a constant $C$ such that $|w(x)| \leq C|| x||^{2}$, then we say that $w$ has quadratic asymptotics and call this the quadratic case.

(2) If $w$ does not have quadratic asymptotics, but

$$
\liminf _{r \downarrow 0} \sup _{\|x\|=r} \frac{|w(x)|}{r^{2}}<\infty
$$

then we say that $w$ has mixed asymptotics and call this the mixed case.

(3) If

$$
\liminf _{r \downarrow 0} \sup _{\|x\|=r} \frac{|w(x)|}{r^{2}}=\infty
$$

then we say that $w$ is strictly superquadratic and call this the strictly superquadratic case.

Any function in the second or third case above will be referred to as superquadratic, which is equivalent to

$$
\lim \sup \sup _{\| \downarrow 0} \frac{|w(x)|}{r^{2}}=\infty
$$

With these definitions we present a method to eliminate the mixed case for two problems which we will now describe. 


\section{Problem 1: An Eigenvalue Optimization Problem Arising in the Study of Composite Membranes}

We want to create a membrane with prescribed shape and mass out of given materials (with different densities) in such a way that the fundamental frequency of the resulting membrane is minimized. In Chanillo et al. (2000a) it is shown that the following mathematical problem is slightly more general than the physical problem just stated. We assume that we are given a domain $\Omega \subset \mathbb{R}^{2}$ which is bounded, connected, and has a Lipschitz boundary, and we are given numbers $\alpha>0$ and $A \in[0,|\Omega|]$ (where $|\Omega|$ denotes the Lebesgue 2-dimensional measure of $\Omega$ ). We let $\chi_{D}$ be the characteristic function of $D$, and $\lambda_{\Omega}(\alpha, D)$ be the minimum eigenvalue of the problem

$$
\begin{aligned}
-\Delta u+\alpha \chi_{D} u & =\lambda u & & \text { in } \Omega \\
u & =0 & & \text { on } \partial \Omega
\end{aligned}
$$

and we define

$$
\Lambda_{\Omega}(\alpha, A):=\inf _{|D|=A, D \subset \Omega} \lambda_{\Omega}(\alpha, D)
$$

Physically speaking, $D$ is where we use one material and $D^{c}$ is where we use the other material. The fact that we are taking a fixed area for $D$ corresponds to our assumption that the mass of the final membrane has been prescribed. Stated very simply the question becomes, "How will $D$ arrange itself to minimize the first eigenvalue?"' If $D$ is a minimizer for Eq. (1.9), and $u$ satisfies Eq. (1.8), then $(u, D)$ is called an optimal pair (or solution). Chanillo and others (see Chanillo et al., 2000a,b) have studied some of the qualitative properties of these solutions. They have shown that if we normalize $u$ by requiring $\|u\|_{L^{2}}=1$ and $u \geq 0$, then the set $D$ has the form $\{x: u(x)<t\}$ for some $t>0$. They also show that $\partial D^{c}$ is real-analytic at any point where $\nabla u \neq 0$.

Because of the analyticity of the boundary at points where $\nabla u \neq 0$, the main obstacle to proving a Hausdorff estimate on the boundary is the lack of a classification of points where the gradient of $u$ vanishes. So we assume that we are at such a point, and to simplify notation we will assume that boundary point is the origin. By taking $w(x):=u(x)-t$, we will have a function which satisfies the structure conditions which we introduce below. (Although we have stated this problem in two dimensions, the only part of our argument which will require us to be in two dimensions will be the exact classification of the various blow ups. The method presented will give nontrivial blow ups for all cases in all dimensions.)

1.1. Remark. In a recent series of papers (see Weiss, 1998, 1999, 2001 for example), Weiss has found many applications of a monotonicity formula which he introduced in Weiss (1999), and there are some similarities between his formula and the use of the estimate (2.3) in this paper. In fact, it seems that the monotonicity formula of Weiss, and the technique which Spruck employed in Spruck (1983) contain the same 
idea expressed in different variables. Within Weiss (2001), Weiss studied the problem

$$
\Delta w=f \chi_{\{u>0\}}-g \chi_{\{u<0\}},
$$

where

$$
f, g \in C^{\alpha}, \quad f \geq 0, \text { and } f+g>0 .
$$

For solutions of (1.10) under the assumptions (1.11) it turns out that there is always a quadratic bound at any point where $w$ and its gradient vanish, so there is no reason to apply the method of this paper to that situation. The solutions of our eigenvalue problem (more precisely $t-u(x)$ ) will satisfy an equation like (1.10), but with $f \geq 0$, and $f+g<0$.

\section{Problem 2: An Obstacle Problem Where the Membrane Can Pass Through the Obstacle}

The second application of our method will be to the following overdetermined problem which has been studied in Caffarelli et al. (2000) and Blank and Shahgholian (2003): We assume that we are given a Hölder continuous function $f$ which satisfies $0<\lambda \leq f(x) \leq \Lambda$ in $B_{1}$ and then a function $w$ and a domain $\Omega \subset B_{1}$ with $0 \in \partial \Omega$ such that $w$ satisfies

$$
\Delta w(x)=\chi_{\Omega}(x) f(x) \quad \text { and } \quad w(x)=\|\nabla w(x)\|=0 \text { for all } x \in \Omega^{c} .
$$

This situation can arise in the obstacle problem when $w$ is taken to be the height of the membrane above the obstacle. In that case, however, $w \geq 0$ and access to the Harnack inequality leads to a quadratic bound. In the problem above, there is no sign assumption on $w$. The no-sign situation can arise in some inverse problems where Dirichlet and Neumann data are prescribed on a piece of the boundary of a domain. For sufficiently regular $f$, Caffarelli et al. show that $w$ must have a quadratic bound (see Caffarelli et al., 2000), but their method which uses the famous AltCaffarelli-Friedman monotonicity formula involves estimates for the Laplacian of a derivative of $w$, and therefore requires at least a weak derivative of $f$.

\section{THE MAIN INGREDIENTS}

Now we turn back to a description of the method we use to eliminate the mixed case. The first ingredient is a theorem which produces a nontrivial blow up in either superquadratic case, and is essentially found in Blank and Shahgholian (2003).

2.1. Theorem (Blank-Shahgholian). Assume that $w$ is superquadratic, that $w(0)=\|\nabla w(0)\|=0$, and that $|\Delta w| \leq C$ in a neighborhood of zero. Then there exists a sequence of radii $\left\{\rho_{j}\right\}$ decreasing to zero, and another sequence $\left\{\gamma_{j}\right\}$ such 
that the rescalings

$$
w_{j}(x):=\gamma_{j} w\left(\rho_{j} x\right)
$$

converge to a nontrivial homogeneous harmonic polynomial of degree 2 on all of $\mathbb{R}^{n}$.

The next ingredient is a change of variables and a theorem which in the quadratic case is essentially due to Spruck. (Our treatment follows Karp and Shahgholian, 2000.) The change of variables is given by

$$
\psi(s, \theta):=\frac{w(r, \theta)}{r^{2}}, \quad \text { with } s:=-\log r
$$

where $(r, \theta)$ are the usual polar coordinates, and the theorem we need is the following:

2.2. Theorem (Spruck). Assume that $w(0)=\|\nabla w(0)\|=0$, that (with $\psi$ defined exactly as in Eq. (2.2))

$$
\int_{S^{n-1}} \int_{1}^{\infty} \psi_{s}^{2} d s d \theta \leq C
$$

that $|\Delta w| \leq C$, and that $\left\{\rho_{j}\right\}$ is a sequence converging to zero such that

$$
\sup _{j} \sup _{x \in \partial B_{\rho_{j}}} \frac{|w(x)|}{\|x\|^{2}} \leq C \quad \text { and } \quad \sup _{j} \sup _{x \in \partial B_{\rho_{j}}} \frac{\|\nabla w(x)\|}{\|x\|} \leq C .
$$

Then a subsequence of the quadratic rescaling

$$
w_{j}(x):=\rho_{j}^{-2} w\left(\rho_{j} x\right)
$$

converges to a function which is homogeneous of degree two.

Before continuing, we which to give a threefold motivation for our change of variables: First, after a separation of variables in polar coordinates, a Laplacian will lead to an Euler equation for the function in $r$. At that point to solve an Euler equation standard ODE textbooks will suggest the change of variables $s:=\log r$ to yield a simple constant coefficient second order ODE, but $s:=-\log r$ will also work. Second, a logarithmic change of variables makes sense for blow up arguments, because when blowing things up, it is necessary to "think dyadically". Finally, the usefulness of the division by $r^{2}$ appears after showing that the $\psi$ which corresponds to the quadratic blow up limit is independent of $s$. In that case the limit is simply $r^{2} \psi(\theta)$.

2.3. Remark. Equation (2.3) is the main structure condition to verify in order to apply the method here, and it holds for both problems outlined above as long as 
Eq. (2.4) holds. In other words, assuming that we are in the mixed case or the quadratically bounded case we will have all the hypotheses we need to apply Spruck's Theorem. (Although the $\rho_{j}$ 's given to us in a mixed case only satisfy the first part of Eq. (2.4), we can use elliptic regularity to get both parts if we are willing to shrink the $\rho_{j}$ 's slightly.) It is also in the proof that Eq. (2.4) will imply Eq. (2.3) that the fact that Euler equations become constant coefficient equations under the change of variables $s:=-\log r$ comes into play very strongly. After a constant coefficient equation is found for $\psi(s, \theta)$ we can multiply by $\psi_{s}(s, \theta)$ and then have many simple techniques at our disposal to help with the estimates. In particular, the whole estimate is reminiscent of standard energy estimates for constant coefficient second order ordinary differential equations.

2.4. Remark. Although the theorems above have already appeared elsewhere in slightly altered forms, we will give their proofs since they have been extended in the present context. In particular, Theorem (2.1) was proven by Blank and Shahgholian for functions which solve the second problem described above, and Theorem (2.2) has so far only been shown and used in cases where there was a quadratic bound on the function in question.

At this point in the mixed case by applying Theorem (2.1) we have an inhomogeneous blow up to a harmonic polynomial along one sequence of radii, and by applying Theorem (2.2) along a quadratically bounded sequence, we will have a quadratic blow up to a function which is homogeneous of degree two. It will turn out that in both problems we are considering, we will be able to classify the quadratic blow up limit, and, not surprisingly, it will have different properties from the inhomogeneous blow up limit obtained along the superquadratic sequence. By taking an interlacing sequence of radii, we will be able to produce a sequence of rescalings (call it $\left\{r_{n}\right\}$ ) where the function is incompatible with either blow up. Now either the function has a quadratic bound along this sequence, i.e.,

$$
\sup _{n} \sup _{x \in \partial B_{r_{n}}} \frac{|w(x)|}{\|x\|^{2}}<\infty
$$

in which case we can use Theorem (2.2) to come to a contradiction, or there exists a subsequence of the $\left\{r_{n}\right\}$ (which we still call $\left\{r_{n}\right\}$ ) such that

$$
\lim _{n \rightarrow \infty} \sup _{x \in \partial B_{r_{n}}} \frac{|w(x)|}{\|x\|^{2}}=\infty
$$

It is for this case where we would like to do an inhomogeneous blow up to come to a contradiction, but we cannot apply Theorem (2.1) because that theorem does not allow for an arbitrary choice of the radii. In other words, the $\left\{r_{n}\right\}$ of the interlacing radii are not necessarily the $\left\{\rho_{n}\right\}$ given by that theorem. The following theorem is the final ingredient of the method to eliminate the mixed case, and the main theorem of this paper. 
2.5. Theorem (Nontrivial Inhomogeneous Blow Up). Assume that $\Delta w \in L^{\infty}$, that $w(0)=\|\nabla w(0)\|=0$, and that with the definition of $\psi$ as above we satisfy the structure condition:

$$
\int_{S^{n-1}} \int_{0}^{\infty} \psi_{s}^{2} d s d \theta \leq C
$$

Finally, assume that a sequence $\left\{t_{j}\right\}$ is given which satisfies

$$
\lim _{j \rightarrow \infty} \int_{B_{1}}\left(\frac{w\left(t_{j} x\right)}{t_{j}^{2}}\right)^{2} d x=+\infty .
$$

Then there exists a subsequence of the $t_{j}$ (still called $t_{j}$ ), and a sequence $\ell_{j} \rightarrow \infty$ such that the rescalings $w_{j}(x):=w\left(t_{j} x\right) /\left(t_{j}^{2} \ell_{j}\right)$ converge to a nontrivial harmonic polynomial of degree two.

2.6. Remark. The sudden switch from superquadratic behavior as we have defined it above to a superquadratic behavior in an $L^{2}$ norm sense is harmless in the applications we give because of elliptic regularity theory. (If supremums become large, then $L^{2}$ norms have to become large.)

\section{THE PROOFS OF THE THREE MAIN THEOREMS}

\section{Proof of Theorem (2.1)}

We follow the proof of compactness given in Blank and Shahgholian (2003). By rescaling we can assume without loss of generality that $|\Delta w| \leq C$ on $B_{2}$. We define the following notation:

$$
S_{j}:=\sup _{x \in B_{2^{-j}}}|w(x)| \quad \text { and } \quad \tilde{S}_{r}:=\sup _{x \in B_{r}}|w(x)| .
$$

Now if there exists a constant $\gamma$ such that for all $j$ we have

$$
S_{j+1} \leq \max \left\{\frac{\gamma 2^{-2 j}}{4}, \frac{S_{j}}{4^{1}}, \ldots, \frac{S_{0}}{4^{j+1}}\right\}
$$

then induction leads to a quadratic bound on $w$ which contradicts our assumptions. Therefore we can assume that there exists a sequence of integers $\left\{k_{j}\right\}$ such that

$$
S_{k_{j}+1}>\max \left\{\frac{j 2^{-2 k_{j}}}{4}, \frac{S_{k_{j}}}{4^{1}}, \ldots, \frac{S_{0}}{4^{k_{j}+1}}\right\}
$$


Since $w \in C^{0}\left(\overline{B_{1}}\right)$, we must have $k_{j} \rightarrow \infty$. Now define

$$
w_{j}(x):=\frac{w\left(2^{-k_{j}} x\right)}{S_{k_{j}+1}},
$$

and note that in $B_{2^{k_{j}}}$ we have

$$
\left|\Delta w_{j}\right| \leq \frac{C}{j} \rightarrow 0
$$

On the other hand, in $B_{2^{m}}$ (for $0 \leq m \leq k_{j}$ ) we have

$$
\left|w_{j}(x)\right| \leq \frac{S_{k_{j}-m}}{S_{k_{j}+1}} \leq 4\left(2^{m}\right)^{2}
$$

so for $\|x\| \geq 1$ we have $\left|w_{j}(x)\right| \leq C|| x \|^{2}$. Because we know that $\left\|w_{j}\right\|_{L^{\infty}\left(B_{1 / 2}\right)}=1$, we can conclude that $w_{j}$ converges to a nontrivial harmonic polynomial of degree 2 which vanishes with its gradient at the origin. Now we can take the $\rho_{j}$ in the statement of the theorem to be $2^{-k_{j}}$ and we can take the $\gamma_{j}$ to be the reciprocal of $S_{k_{j}+1}$.

\section{Proof of Theorem (2.2)}

We follow the treatment in Karp and Shahgholian (2000) and make the following definitions:

$$
\begin{aligned}
& w_{j}(r, \theta):=\rho_{j}^{-2} w\left(\rho_{j} r, \theta\right)=\frac{w\left(\rho_{j} r, \theta\right)}{\rho_{j}^{2}} \\
& \psi_{j}(s, \theta):=\frac{w_{j}(r, \theta)}{r^{2}}=\frac{w\left(\rho_{j} r, \theta\right)}{\left(\rho_{j} r\right)^{2}} .
\end{aligned}
$$

So the $w_{j}$ 's are the quadratic rescalings of the original function $w$, and the $\psi_{j}$ 's are just the corresponding $\psi$ 's. Because the Laplacians of $w_{j}$ are bounded independently of $j$, and because the $w_{j}$ are bounded uniformly on $\partial B_{1}$, we have $C^{1, \beta}$ bounds on every compact set. So, by passing to a subsequence if necessary, we can assume that the sequence $\rho_{j}$ has been chosen so that $w_{j} \rightarrow w_{\infty}$ uniformly in $C^{1, \beta}$ on every compact set.

Convergence of the $w_{j}$ to $w_{\infty}$ implies

$$
\frac{\partial \psi_{j}}{\partial s} \rightarrow \frac{\partial \psi_{\infty}}{\partial s} \quad \text { in } L^{2}\left([0, N] \times S^{n-1}\right)
$$


for any $N<\infty$, where $\psi_{\infty}(s, \theta):=w_{\infty}(r, \theta) /\left(r^{2}\right)$. Now by the bounds and convergence just mentioned along with the identity $\psi_{j}(s, \theta)=\psi\left(s-\log \rho_{j}, \theta\right)$, we have

$$
\begin{aligned}
\int_{S^{n-1}} \int_{0}^{N}\left(\frac{\partial \psi_{\infty}}{\partial s}\right)^{2} d s d \theta & =\lim _{j \rightarrow \infty} \int_{S^{n-1}} \int_{0}^{N}\left(\frac{\partial \psi_{j}}{\partial s}\right)^{2} d s d \theta \\
& =\lim _{j \rightarrow \infty} \int_{S^{n-1}} \int_{-\log \rho_{j}}^{N-\log \rho_{j}}\left(\frac{\partial \psi}{\partial s}\right)^{2} d s d \theta=0
\end{aligned}
$$

by using our structure condition, Eq. (2.3). Thus $\psi_{\infty}(s, \theta)=\psi_{\infty}(\theta)$ and $w_{\infty}(s, \theta)=s^{2} \psi_{\infty}(\theta)$.

\section{Proof of Theorem (2.5)}

3.1. Remark. The crucial point here is the nontrivial nature of the blowup. Obviously the $\ell_{j}$ will be chosen to normalize an appropriate norm of the $w_{j}$, and by doing this type of normalization we will have convergence to a harmonic function. On the other hand, by again considering the functions $r^{n} \sin n \theta$ on the disk, it is easy to produce a sequence of harmonic functions whose $L^{\infty}$ norm is always 1 (or whose $L^{2}$ norm is always 1 ), but which converge to 0 uniformly in any compact subset of the disk. In particular, the situation seems to be reminiscent of some of the problems treated with the theory of concentrated compactness. In this setting, however, there is no use of any variational structure.

We choose the constants $\ell_{j}$ to normalize the $L^{2}$ norm of our rescalings on the ball $B_{1}$. We also take a subsequence to ensure that the $\ell_{j}$ 's are increasing and converge to infinity. We can also assume by taking a further subsequence that the $w_{j}$ now converge to a harmonic function $W$ on the unit disk. The convergence will be uniform in $C^{1, \alpha}$ for every $\alpha<1$ on every compact subset of $B_{1}$. Now to prove things by contradiction, we will assume that $W \equiv 0$ in $B_{1}$.

With the $L^{2}$ norms of our $w_{j}$ 's normalized to be one, it is clear that in order for the $w_{j}$ to be converging to 0 , the mass of the $L^{2}$ norm must be moving toward $\partial B_{1}$, and this concentration along with the normalization, force the supremum of $\left|w_{j}\right|$ on $\partial B_{1}$ to become arbitrarily large. Now the function $w_{j}$ satisfies a Poisson equation on $B_{3}$ with a Laplacian bounded by a number going to zero as $j \rightarrow \infty$. In particular, by Krylov-Safonov estimates (see Theorem 9.20 of Gilbarg and Trudinger, 1983), the $L^{2}$ norm of $w_{j}$ on $B_{3}$ is getting arbitrarily large (see Figure 1).

Now we consider our change of variables again. As long as we have $r$ bounded away from 0 and $\infty$, the $L^{2}$ norms of $w_{j}$ and the corresponding $\psi_{j}$ will be comparable. Our definitions of the $w_{j}$ and of the $\psi_{j}$ now include the extra $\ell_{j}$ term from the statement of the theorem:

$$
\begin{aligned}
& w_{j}(r, \theta):=\frac{w\left(t_{j} r, \theta\right)}{t_{j}^{2} \ell_{j}}=\frac{w\left(t_{j} r, \theta\right)}{t_{j}^{2} \ell_{j}} \\
& \psi_{j}(s, \theta):=\frac{w_{j}(r, \theta)}{r^{2}}=\frac{w\left(t_{j} r, \theta\right)}{\left(t_{j} r\right)^{2} \ell_{j}}
\end{aligned}
$$




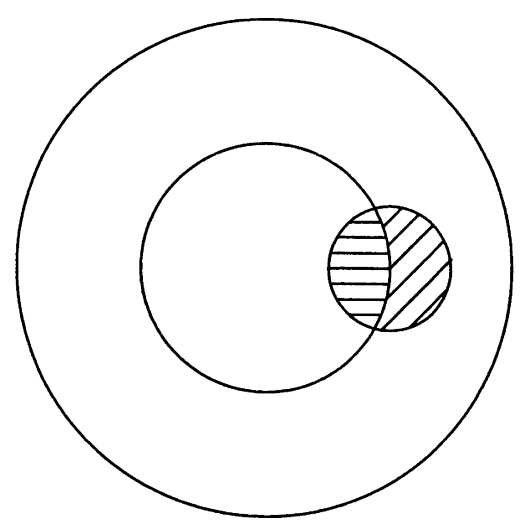

Figure 1. The concentric balls are centered at the origin and have radii equal to one and three. (The figure is not drawn to scale.) The center of the other ball lies on the sphere of radius one, and is a place where $\left|w_{j}\right|$ becomes very large. By our normalizations we know that the $L^{2}$ norm of $w_{j}$ is bounded by 1 on the horizontally striped region, but by elliptic regularity we know that the $L^{2}$ norm is very large on the diagonally striped region.

For our situation now we know that

$$
\int_{S^{n-1}} \int_{0}^{1} \psi_{j}^{2} d s d \theta
$$

is as small as we like, and

$$
\int_{S^{n-1}} \int_{-1}^{0} \psi_{j}^{2} d s d \theta
$$

is as large as we like. On the other hand, by using Eq. (2.3) and by taking $j$ sufficiently large we have an arbitrarily small bound on

$$
\int_{S^{n-1}} \int_{-1}^{1}\left(\frac{\partial \psi_{j}}{\partial s}\right)^{2} d s d \theta
$$

(The extra $\ell_{j}$ term only helps us here.) Now the usual Sobolev Inequalities do not apply, since they require a bound on the entire gradient of $\psi$ which we do not have. On the other hand, for Poincaré's Inequality, if the geometry of the domain is cooperative, then we can get away with an integral estimate of only one partial derivative, as we will prove in the next lemma. Except for that lemma we're done. (The fact that our nontrivial blowup is a polynomial of degree two, follows from the estimates we have on $\psi_{s}$.)

To simplify things slightly, we will state and prove our lemma in a rectangular case, but it is clear that nothing changes in the radial case. 
3.2. Lemma (Poincaré' Type Result). Let $D:=[-1,1]^{n}$, and let $u \in W^{1,2}(D)$. Let $D^{+}:=D \cap\left\{x_{n}>0\right\}$, and $D^{-}:=D \cap\left\{x_{n}<0\right\}$. If

$$
\|u\|_{L^{2}\left(D^{-}\right)} \leq 1
$$

and

$$
\left\|\frac{\partial u}{\partial x_{n}}\right\|_{L^{2}(D)} \leq 1
$$

then

$$
\|u\|_{L^{2}\left(D^{+}\right)} \leq 4
$$

Proof. By approximation, we can assume that $u$ is smooth. Let $x=\left(x^{\prime}, x_{n}\right) \in D^{+}$. Then

$$
u(x)=u\left(x^{\prime}, x_{n}-1\right)+\int_{x_{n}-1}^{x_{n}} \frac{\partial u}{\partial x_{n}}\left(x^{\prime}, t\right) d t
$$

Hence

$$
|u(x)| \leq\left|u\left(x^{\prime}, x_{n}-1\right)\right|+\left(\int_{x_{n}-1}^{x_{n}}\left|\frac{\partial u}{\partial x_{n}}\left(x^{\prime}, t\right)\right|^{2} d t\right)^{1 / 2}
$$

and so,

$$
|u(x)|^{2} \leq 2\left|u\left(x^{\prime}, x_{n}-1\right)\right|^{2}+2 \int_{x_{n}-1}^{x_{n}}\left|\frac{\partial u}{\partial x_{n}}\left(x^{\prime}, t\right)\right|^{2} d t .
$$

Integration over $x^{\prime}$ yields

$$
\int_{[-1,1]^{n-1}}|u(x)|^{2} d x^{\prime} \leq 2 \int_{[-1,1]^{n-1}}\left|u\left(x^{\prime}, x_{n}-1\right)\right|^{2} d x^{\prime}+2 \int_{D}\left|\frac{\partial u}{\partial x_{n}}(x)\right|^{2} d x .
$$

Finally, we integrate $x_{n}$ over $[0,1]$ to get

$$
\int_{D^{+}}|u(x)|^{2} d x \leq 2 \int_{D^{-}}|u(x)|^{2} d x+2 \int_{D}\left|\frac{\partial u}{\partial x_{n}}(x)\right|^{2} d x .
$$




\section{VERIFYING THE HYPOTHESES}

\section{Verifying the Hypotheses for Our Eigenvalue Optimization Problem}

Now we turn to the verification of the hypotheses we need in order to apply our method to our model problems. For our eigenvalue problem, the $w$ we introduced before will satisfy:

$$
\Delta w(x)=\left(\alpha \chi_{w \leq 0}(x)-\lambda\right)(w(x)+t)
$$

and $w(0)=\|\nabla w(0)\|=0$. We need to verify the structure condition (2.3) holds in the mixed case. We start with a sequence $\rho_{j} \downarrow 0$ such that

$$
\sup _{j} \sup _{x \in \partial B_{\rho_{j}}} \frac{|w(x)|}{\|x\|^{2}} \text { is bounded. }
$$

By using elliptic regularity and replacing each $\rho_{j}$ with $\rho_{j} / 2$ we can guarantee that not only does Eq. (4.2) hold, but that we also have

$$
\sup _{j} \sup _{x \in \partial B_{\rho_{j}}} \frac{\|\nabla w(x)\|}{\|x\|} \text { is bounded }
$$

which is the other half of Eq. (2.4). We define

$$
\psi(s, \theta):=\frac{w(r, \theta)}{r^{2}}, \quad \text { with } s:=-\log r
$$

as before. Note that

$$
\psi_{s}(s, \theta)=2 \frac{w(r, \theta)}{r^{2}}-\frac{w_{r}(r, \theta)}{r},
$$

and

$$
\psi_{s s}(s, \theta)=4 \frac{w(r, \theta)}{r^{2}}-3 \frac{w_{r}(r, \theta)}{r}+w_{r r}(r, \theta) .
$$

A simple computation shows

$$
\psi_{s s}-4 \psi_{s}+4 \psi+\psi_{\theta \theta}=\Delta w=\left(\alpha \chi_{\{w \leq 0\}}-\lambda\right)(w+t) .
$$

We multiply this equation by $\psi_{s}$ and integrate over $B_{T} \backslash B_{1}$ with measure $d s d \theta$ to get

$$
\begin{gathered}
\left.\frac{1}{2} \int_{0}^{2 \pi}\left[\psi_{s}^{2}+4 \psi^{2}-\psi_{\theta}^{2}\right]\right|_{1} ^{T} d \theta-4 \int_{0}^{2 \pi} \int_{1}^{T} \psi_{s}^{2} d s d \theta \\
=\int_{0}^{2 \pi} \int_{1}^{T}\left(\alpha \chi_{\{\psi \leq 0\}}-\lambda\right)(w+t) \psi_{s} d s d \theta .
\end{gathered}
$$


Using Eq. (4.5) and our bounds on $\left|\psi_{s}\right|$ and $|\psi|$ we get

$$
\begin{aligned}
4 \int_{0}^{2 \pi} \int_{1}^{T} \psi_{s}^{2} d s d \theta & \leq 4 \int_{0}^{2 \pi} \int_{1}^{T} \psi_{s}^{2} d s d \theta+\frac{1}{2} \int_{0}^{2 \pi} \psi_{\theta}^{2}(T, \theta) d \theta \\
& \leq C-\int_{0}^{2 \pi} \int_{1}^{T}\left(\alpha \chi_{\{\psi \leq 0\}}-\lambda\right)(w+t) \psi_{s} d s d \theta \\
& \leq C-\int_{0}^{2 \pi} \int_{1}^{T}\left(\alpha \chi_{\{\psi \leq 0\}}-\lambda\right) w \psi_{s} d s d \theta
\end{aligned}
$$

for $T$ equal to $-\log \left(\rho_{j}\right)$ where we have used the fundamental theorem of calculus. (If we only use Young's inequality, then our estimates will depend on T.) Because $w \in C^{1, \beta}$ and because $w$ and its gradient vanish at the origin, we have $|w(s, \theta)| \leq C e^{-(1+\beta) s}$, and $\left|w_{r}(s, \theta)\right| \leq e^{-\beta s}$, so

$$
\left\|\int_{0}^{2 \pi} \int_{1}^{T}\left(\alpha \chi_{\{\psi \leq 0\}}-\lambda\right) w \psi_{s} d s d \theta\right\| \leq C \int_{1}^{\infty}(\alpha+\lambda) e^{-(1+\beta) s} e^{(1-\beta) s} d s d \theta \leq C
$$

which proves Eq. (2.3). At this point we can apply all of our theorems in the mixed case. We turn to the classifications in dimension two.

By assuming that we are in dimension two, and by a rotation of coordinates if necessary, we know that the inhomogeneous blow ups we get are a multiple of $x^{2}-y^{2}$, and therefore the density of our positivity set along the set of radii which lead to this blow up must equal one half. On the other hand, we can apply Theorem (2.2) along a sequence of radii with a quadratic bound, and then the limiting function $w_{\infty}(r, \theta)$ satisfies:

(a) $w_{\infty}(r, \theta)=r^{2} f(\theta) \in C^{1, \beta}\left(\mathbb{R}^{2}\right)$

(b) There exist constants $\gamma<\mu<0$ such that

$$
\begin{aligned}
& f^{\prime \prime}(\theta)+4 f(\theta)= \begin{cases}4 \gamma & f(\theta)>0 \\
4 \mu & f(\theta)<0\end{cases} \\
& \mu=(\alpha-\lambda) t / 4, \text { and } \gamma=-\lambda t / 4 .
\end{aligned}
$$

4.1. Remark. The preceding equation follows quickly from the fact that $w_{\infty}$ satisfies the equation

$$
\Delta w_{\infty}(x)=\left(\alpha \chi_{\left\{w_{\infty} \leq 0\right\}}-\lambda\right) t
$$

To verify this equation for $w_{\infty}$, it is easiest to consider what happens to the Laplacian of $w$ when Eq. (4.1) is rescaled. Equation (4.1) can be written

$$
\Delta w(x)=\left(\alpha \chi_{\{w \leq 0\}}-\lambda\right) f(x)
$$


where $f(x) \in C^{0}$ with $f(0)=t$. (By using only these properties of $f$ this result is much more transparent than if we focus on the additional structure we have for $f$. In other words, ignore the fact that $f(x)$ happens to equal $w(x)+t$ when deriving this equation.)

After rotating the coordinates, we can assume that there are constants $0<\tilde{\theta}<\theta_{\text {end }}$, such that

(a) $0=f(0)=f(\tilde{\theta})=f\left(\theta_{\text {end }}\right)$

(b) $f(\theta)$ is positive in $(0, \tilde{\theta})$ and negative in $\left(\tilde{\theta}, \theta_{\text {end }}\right)$.

Let $I_{1}:=[0, \tilde{\theta}]$, and $I_{2}:=\left[\tilde{\theta}, \theta_{\text {end }}\right]$. Now there are constants $C_{-}, C_{+}, D_{-}$, and $D_{+}$ such that

$$
f(\theta)= \begin{cases}C_{+} \sin \left(2 \theta+D_{+}\right)+\gamma & \text { in } I_{1} \\ C_{-} \sin \left(2 \theta+D_{-}\right)+\mu & \text { in } I_{2}\end{cases}
$$

We can assume that $C_{ \pm}>0$, and because $\gamma<\mu<0$, we know that $0<\left|I_{1}\right|<\pi / 2$ and $\pi / 2<\left|I_{2}\right|<\pi$. By the symmetry of the sine function, we also know that $0<f^{\prime}(0)=-f^{\prime}(\tilde{\theta})=f^{\prime}\left(\theta_{\text {end }}\right)$. By uniqueness of solutions of ordinary differential equations we see that the shape of the "positive hump" of $f$ which follows $\theta_{\text {end }}$, will be identical to the shape of the positive hump on $I_{1}$. Thus, we are led to a rotational symmetry, which by periodicity and our estimates of $\left|I_{j}\right|$ force

$$
\theta_{\text {end }}=\pi \quad \text { or } \quad \theta_{\text {end }}=2 \pi / 3 \text {. }
$$

4.2. Lemma $\left(\theta_{\text {end }}=2 \pi / 3\right)$.

Proof. We will assume that $\theta_{\text {end }}=\pi$. Because $|\sin x| \leq 1$, we have $C_{-}>|\mu|$ and $C_{+}>|\gamma|$. Now we write down the things which follow from the compatibility conditions and our assumptions, and we stress that our arcsine function will follow the usual convention and always assume values between $-\pi / 2$ and $\pi / 2$. (In other words, we have worked out all of the potential problems of different "branches" of the arcsine function in the computations below, and in so doing we will always assume that $D_{+}$and $D_{-}$are equal to whatever are the most convenient values for us.)

(i) $f(0)=0$, so after assuming that $0 \leq D_{+} \leq \pi / 2$, we get

$$
D_{+}=\arcsin \left(-\gamma / C_{+}\right)
$$

and then

$$
\tilde{\theta}=(\pi / 2)+\arcsin \left(\gamma / C_{+}\right) .
$$

(ii) $f(\tilde{\theta})=0$, so

$D_{-}=\arcsin \left(\mu / C_{-}\right)-2 \arcsin \left(\gamma / C_{+}\right)$.

(The possibility $D_{-}=\pi-\arcsin \left(\mu / C_{-}\right)-2 \arcsin \left(\gamma / C_{+}\right)$can be eliminated.) 
(iii) $f^{\prime}(\tilde{\theta})$ exists, so

$2 C_{+} \cos \left(\pi+2 \arcsin \left(\gamma / C_{+}\right)+D_{+}\right)=2 C_{-} \cos \left(\pi+2 \arcsin \left(\gamma / C_{+}\right)+D_{-}\right)$,

which leads to

$C_{+}^{2}-\gamma^{2}=C_{-}^{2}-\mu^{2}$

(iv) $f(\pi)=0$, so

$C_{-} \sin \left(D_{-}\right)+\mu=0$

which leads to

$$
\frac{\mu^{2}}{C_{-}^{2}}=\frac{\gamma^{2}}{C_{+}^{2}}
$$

Now by using (iii) and (iv), we get

$$
C_{+}^{2}\left(1-\frac{\gamma^{2}}{C_{+}^{2}}\right)=C_{+}^{2}-\gamma^{2}=C_{-}^{2}-\mu^{2}=C_{-}^{2}-C_{-}^{2} \frac{\gamma^{2}}{C_{+}^{2}}=C_{-}^{2}\left(1-\frac{\gamma^{2}}{C_{+}^{2}}\right)
$$

which implies $C_{+}=C_{-}$, which in turn implies $\mu=\gamma$, which is a contradiction.

By using the estimate $\pi / 2 \leq\left|I_{2}\right|$, combined with the last lemma, we see that the density of the positivity set in the quadratic limit is less than $25 \%$ (see Fig. 2).

It is now a simple matter to produce an interlacing sequence such that the density of the positivity set along this sequence is not compatible with either blow up. Then by applying either Theorem (2.5) or Theorem (2.2) according to the asymptotics on the interlacing sequence we have a contradiction. We summarize this information in the following theorem.

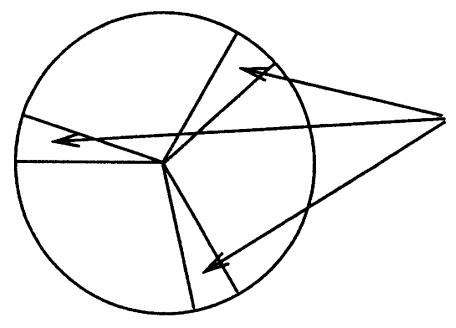

Regions where the

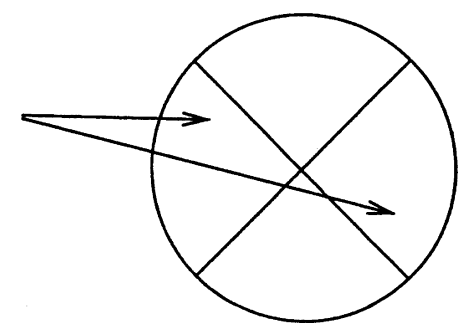

Figure 2. The positivity set in the quadratic limit. 
4.3. Theorem (Asymptotics for the Composite Membrane Problem). Assume $w$ satisfies (4.1) in dimension 2 and assume that $w(0)=\|\nabla w(0)\|=0$. Then either $w$ has a quadratic bound, or $w$ is strictly superquadratic.

\section{Verifying the Hypotheses for Our "No Sign" Obstacle Problem}

For the second problem, the proof that in the mixed case the structure condition (2.3) will hold is very similar to the proof for the eigenvalue problem, so we will omit it. The proof applies to problems where the $f$ is only Hölder continuous, which is a situation where it does not seem possible to apply the monotonicity formula of Caffarelli et al. (2002) to a derivative of $w$. For simplicity of notation we will assume henceforth without loss of generality that $f(0)=2$.

The classification work which we would need to do has been done already in a paper by Caffarelli et al. (2000). Their paper shows that quadratic blow up limits must converge to either a rotation of

$$
\left(x_{n}^{+}\right)^{2}
$$

or to a rotation of a function of the form

$$
\sum_{j=1}^{n} \alpha_{j} x_{j}^{2} \quad \text { where } \sum_{j=1}^{n} \alpha_{j}=1
$$

On the other hand, inhomogeneous blow ups must be of the form (after rotation if necessary)

$$
\sum_{j=1}^{n} \beta_{j} x_{j}^{2} \quad \text { where } \sum_{j=1}^{n} \beta_{j}=0 \text { and } \beta_{n} \neq 0 .
$$

Note that first derivatives of either of the functions of the form (4.12) or (4.13) are odd functions, unlike the situation in the case where the blow up is $\left(x_{n}^{+}\right)^{2}$. We will use this idea, along with a weak thickness condition which ensures the existence of blow ups of the form $\left(x_{n}^{+}\right)^{2}$ to derive a contradiction. As in Caffarelli (1977) we introduce the minimum diameter of a set $S \in \mathbb{R}^{n}$ which we denote by m.d.(S) and which is the infimum of the distances between parallel hyperplanes which enclose $S$. Now if

$$
\inf _{0<r \leq 1 / 2} \frac{m \cdot d \cdot\left(\Omega^{c} \cap B_{r}\right)}{r} \geq \epsilon>0
$$

for any fixed $\epsilon>0$, then it is shown in Blank and Shahgholian (2003) that there exists a $C=C(\epsilon)$ such that $|w(x)| \leq C\|x\|^{2}$ in $B_{1 / 2}$. (In fact there is a slightly stronger result in Blank and Shahgholian (2003).) Now we have the following statement when the infimum is replaced by a limsup. 
4.4. Theorem (Asymptotics for the No-Sign Obstacle Problem). Assume that $w$ is a solution of our no-sign obstacle problem (i.e., it satisfies (1.12)), and assume that

$$
\underset{r \downarrow 0}{\limsup } \frac{m \cdot d \cdot\left(\Omega^{c} \cap B_{r}\right)}{r}>0,
$$

then $w$ cannot have mixed asymptotics at zero.

Proof. Let $\left\{q_{j}\right\} \downarrow 0$ denote the superquadratic sequence given by Theorem (2.1) which gives an inhomogeneous blow up to a nontrivial harmonic polynomial which is homogeneous of degree two. By our assumption (4.15) there will be a sequence $\left\{r_{j}\right\} \downarrow 0$ such that the minimum diameters of the zero set for the corresponding rescalings stay greater than a positive number. By invoking Theorem (2.5) and the classification results of Caffarelli et al. (2000) we come to a contradiction if $\left\{r_{j}\right\}$ is not a quadratically bounded sequence with a blow up to a rotation of $\left(x_{n}^{+}\right)^{2}$.

Now let $\gamma(n):=\left\|\left(x_{n}^{+}\right)^{2}\right\|_{L^{2}\left(B_{1}\right)}$, and make the following definition:

$$
v_{\epsilon}(x):=t_{\epsilon} w(\epsilon x)
$$

where $t_{\epsilon}$ is defined by the condition that

$$
\left\|v_{\epsilon}(x)\right\|_{L^{2}\left(B_{1}\right)} \equiv \gamma(n)
$$

Next let $V_{\epsilon}(x)$ be a rotation of $v_{\epsilon}(x)$ which maximizes

$$
\Theta(\epsilon)=\Theta\left(V_{\epsilon}(x)\right):=\int_{B_{1 / 2}} \frac{\partial V_{\epsilon}(x)}{\partial e_{n}} d x
$$

$\Theta(\epsilon)$ is a continuous function of $\epsilon$ and if we set $\Gamma(n):=\int_{B_{1 / 2}} 2 x_{n}^{+} d x>0$, then we know by using $C^{1, \alpha}$ convergence that

$$
\lim _{j \rightarrow \infty} \Theta\left(r_{j}\right)=\Gamma(n)>0 \text { and } \lim _{j \rightarrow \infty} \Theta\left(q_{j}\right)=0
$$

Let $\left\{s_{j}\right\}$ be a sequence such that $\Theta\left(s_{j}\right)=\Gamma(n) / 2$. After taking a subsequence of the $\left\{s_{j}\right\}$ (which we will still call $\left\{s_{j}\right\}$ ) we can be sure that either the $L^{2}$ norms of the functions given by $w_{j}(x):=s_{j}^{-2} w\left(s_{j} x\right)$ are bounded, or they go to infinity.

If these norms are unbounded, then by using Theorem (2.5) we get convergence of the $V_{s_{j}}(x)$ to a harmonic polynomial which is homogeneous of degree two, and in particular we would have $\Theta\left(s_{j}\right) \rightarrow 0$ which is a contradiction. (The normalization within Theorem (2.5) fixes the $L^{2}$ norms, and it is therefore comparable to the normalization which gives us the $V_{s_{j}}(x)$ from the $w_{j}(x)$.) 
On the other hand, if the $L^{2}$ norms stay bounded, then after defining $W_{j}(x)$ to be the rotation of $w_{j}(x)$ which maximizes

$$
\widetilde{\Theta}(j)=\widetilde{\Theta}\left(W_{j}(x)\right):=\int_{B_{1 / 2}} \frac{\partial W_{j}(x)}{\partial e_{n}} d x,
$$

and then taking a subsequence if necessary, we can use the classification results of Caffarelli et al. (2000) to get convergence of the sequence $\left\{W_{j}(x)\right\}$ to either $\left(x_{n}^{+}\right)^{2}$, or a polynomial of the form given by (4.12). In the first case, $\left\|W_{j}\right\|_{L^{2}\left(B_{1}\right)} \rightarrow \gamma(n)$. Recall that $\gamma(n)=\left\|v_{\epsilon}(x)\right\|_{L^{2}\left(B_{1}\right)}=\left\|V_{\epsilon}(x)\right\|_{L^{2}\left(B_{1}\right)}$, so in particular $\left[\widetilde{\boldsymbol{\Theta}}(j)-\Theta\left(s_{j}\right)\right]$ converges to 0 , and therefore both $\widetilde{\Theta}(j)$ and $\Theta\left(s_{j}\right)$ converge to $\Gamma(n) \neq \Gamma(n) / 2$. In the second case, by using the convergence to a function of the form given by (4.12) and by using the fact that we have bounded $L^{2}$ norms we have $\widetilde{\Theta}(j) \rightarrow 0$. By the boundedness of the $L^{2}$ norms our quadratic rescalings, $\Theta\left(s_{j}\right)$ and $\widetilde{\Theta}(j)$ are comparable, so $\Theta\left(s_{j}\right)$ converges to $0 \neq \Gamma(n) / 2$.

\section{ACKNOWLEDGMENTS}

I would like to thank Sagun Chanillo for introducing me to the eigenvalue problem and for his help. I would like to thank Cameron Connell and Derek Hansen for insightful conversations, and I would like to thank an anonymous referee for comments leading to a clarification of the proof of Theorem (4.4).

\section{REFERENCES}

Alt, H. W., Caffarelli, L. A., Friedman, A. (1984). Variational problems with two phases and their free boundaries. Trans. Am. Math. Soc. 282(2):431-461.

Blank, I. (2001). Sharp results for the regularity and stability of the free boundary in the obstacle problem. Indiana Univ. Math. J. 50(3):1077-1112.

Blank, I., Shahgholian, H. (2003). Boundary regularity and compactness for overdetermined problems. Ann. Scuola. Norm. Sup. Pisa. Cl. Sci. 2(4):787-802.

Brézis, H., Kinderlehrer, D. (1973/1974). The smoothness of solutions to nonlinear variational inequalities. Indiana Univ. Math. J. 23:831-844.

Caffarelli, L. A. (1977). The regularity of free boundaries in higher dimensions. Acta Math. 139(3-4):155-184.

Caffarelli, L. A. (1998). The obstacle problem revisited. J. Fourier Anal. Appl. 4(4-5):383-402.

Caffarelli, L. A., Kinderlehrer, D. (1980). Potential methods in variational inequalities. J. Analyse Math. 37:285-295.

Caffarelli, L. A., Karp, L., Shahgholian, H. (2000). Regularity of a free boundary with application to the Pompeiu problem. Ann. Math. (2) 151(1):269-292.

Caffarelli, L. A., Jerison, D., Kenig, C. E. (2002). Some new monotonicity theorems with applications to free boundary problems. Ann. Math. (2) 155(2):369-404. 
Chanillo, S., Grieser, D., Imai, M., Kurata, K., Ohnishi, I. (2000). Symmetry breaking and other phenomena in the optimization of eigenvalues for composite membranes. Comm. Math. Phys. 214(2):315-337.

Chanillo, S., Grieser, D., Kurata, K. (2000). The free boundary problem in the optimization of composite membranes. Differential geometric methods in the control of partial differential equations. Contemp. Math. 268:61-81.

Gilbarg, D., Trudinger, N. S. (1983). Elliptic Partial Differential Equations of Second Order. 2nd ed. Springer-Verlag.

Karp, L., Shahgholian, H. (2000). Regularity of a free boundary at the infinity point. Comm. P.D.E. 25(11-12):2055-2086.

Spruck, J. (1983). Uniqueness in diffusion model of population biology. Comm. P.D.E. 8:1605-1620.

Weiss, G. S. (1998). Partial regularity for weak solutions of an elliptic free boundary problem. Comm. P.D.E. 23:439-455.

Weiss, G. S. (1999). A homogeneity improvement approach to the obstacle problem. Invent. Math. 138:23-50.

Weiss, G. S. (2001). An obstacle-problem-like equation with two phases: Pointwise regularity of the solution and an estimate of the Hausdorff dimension of the free boundary. Interfaces and Free Boundaries 3:121-128.

Received May 2003

Accepted April 2004 


\section{Request Permission or Order Reprints Instantly!}

Interested in copying and sharing this article? In most cases, U.S. Copyright Law requires that you get permission from the article's rightsholder before using copyrighted content.

All information and materials found in this article, including but not limited to text, trademarks, patents, logos, graphics and images (the "Materials"), are the copyrighted works and other forms of intellectual property of Marcel Dekker, Inc., or its licensors. All rights not expressly granted are reserved.

Get permission to lawfully reproduce and distribute the Materials or order reprints quickly and painlessly. Simply click on the "Request Permission/ Order Reprints" link below and follow the instructions. Visit the U.S. Copyright Office for information on Fair Use limitations of U.S. copyright law. Please refer to The Association of American Publishers' (AAP) website for guidelines on Fair Use in the Classroom.

The Materials are for your personal use only and cannot be reformatted, reposted, resold or distributed by electronic means or otherwise without permission from Marcel Dekker, Inc. Marcel Dekker, Inc. grants you the limited right to display the Materials only on your personal computer or personal wireless device, and to copy and download single copies of such Materials provided that any copyright, trademark or other notice appearing on such Materials is also retained by, displayed, copied or downloaded as part of the Materials and is not removed or obscured, and provided you do not edit, modify, alter or enhance the Materials. Please refer to our Website User Agreement for more details.

\section{Request Permission/Order Reprints}

Reprints of this article can also be ordered at http://www.dekker.com/servlet/product/DOI/101081PDE200033762 